\title{
Expressions of Sphingosine-1-phosphate (S1P) Receptors, Sphingosine Kinases in Malignant Bone and Soft Tissue Tumors, and the Role of Sphingosine Kinase-1 in Growth of MFH Cell Lines
}

\author{
Shin-Ichiro Kishimoto ${ }^{1}$, Toshihiro Akisue ${ }^{1 *}$, Kenta Kishimoto ${ }^{1}$, Hitomi Hara ${ }^{1}$, Masaya Imabori ${ }^{1}$, \\ Yoshiyuki Okada ${ }^{1}$, Naomasa Fukase ${ }^{1}$, Teruya Kawamoto ${ }^{1}$, Ikuo Fujita ${ }^{2}$, Takuya Fujimoto ${ }^{2}$, \\ Masahiro Kurosaka ${ }^{1}$

\footnotetext{
${ }^{1}$ Department of Orthopaedic Surgery, Kobe University Graduate School of Medicine, Kusunoki-cho, Chuo-ku, Kobe, Japan;

${ }^{2}$ Department of Orthopaedic Surgery, Hyogo Cancer Center, Kitaoji-cho, Akashi, Hyogo, Japan.

Email: akisue@med.kobe-u.ac.jp
}

Received June $1^{\text {st }}, 2011$; revised June 21 ${ }^{\text {st }}, 2011$; accepted June $28^{\text {th }}, 2011$.

\begin{abstract}
Sphingolipids are ubiquitous components of cell membranes. Their metabolites ceramide, sphingosine, and sphingosine-1-phosphate $(S 1 P)$ have important physiological functions, including regulation of cell growth and survival. S1P is generated by phosphorylation of sphingosine catalyzed by sphingosine kinase-1 (SPHK1). The purpose of this study is to explore the roles of $S 1 P, S 1 P$ receptors, and sphingosine kinases in malignant musculoskeletal tumors. Twenty-one tumor samples (7 liposarcomas, 3 chondrosarcomas, 6 osteosarcomas, 5 MFH) obtained at open biopsy, and four human MFH cell lines (Nara H, Nara F, TNMY1, GBS-1) were used. We examined the mRNA expression of S1P receptors by RT-PCR, and the expression levels of SPHK by Real-time PCR. We used 4 MFH cell lines to analyze SPHK1 proteins by Western blotting. SPHK1 siRNA was transfected into MFH cell lines by lipofection method. Cell proliferation (control and transfected with siRNA) was assayed using WST-8 (Cell Counting Kit-8) assay. All high grade malignant tumors expressed $S 1 P 1, S 1 P 2, S 1 P 3$ receptors, whereas the expression of $S 1 P 1$ receptor was detected in $50 \%$ of low-grade malignant tumors, $S 1 P 2$ receptor in $30 \%$, and $S 1 P 3$ in $50 \%$. No statistically significant difference was found in the expression level of SPHK1 between high-grade and low-grade malignant tumors by Real-time PCR. By results of Western blotting, proteins of SPHK1 were expressed in all MFH cell lines. In MFH cell lines, transfection with SPHK1 siRNA oligonucleotides resulted in approximately 50 to 80\% suppression of SPHK1 mRNA expression as determined by real-time PCR. Down-regulation of SPHK1 with small interfering RNA significantly reduced SPHK1 protein levels by Western blotting. Knock down of SPHK1 expression significantly decreased cell proliferation of all MFH cells. These results suggest that the expression of $S 1 P$ receptors may play an important role for cell proliferation and may correlate with histologic grade in malignant bone and soft tissue tumors, and that SPHK1 may be one of essential molecules for cell proliferation in MFH cell lines.
\end{abstract}

Keywords: Sarcoma, Sphingosine-1-phosphate, S1P Receptor, Sphingosine Kinase, MIB-1, MFH

\section{Introduction}

It has been widely recognized that sphingolipids are not only structural membrane components, but also the important source of bioactive signaling molecules. Sphingolipid metabolites play important roles in physiological and pathological processes. Ceramide is a precursor of sphingosine-1-phosphate (S1P), and ceramide itself is one of important molecules related to cell-growth arrest and apoptosis, whereas sphingosine-1-phosphate (S1P) is a bioactive lipid that promotes cellular proliferation, migration, survival, cytoskeletal rearrangement, and angiogenesis [1-4]. This suggests that the balance between 
Soft Tissue Tumors, and the Role of Sphingosine Kinase-1 in Growth of MFH Cell Lines

levels of S1P versus ceramide act as a switch that determines cell survival or death. S1P is originally thought to act as an intracellular second messenger, it is now widely accepted as a ligand for a family of G-protein-coupled receptors (GPCRs), now called S1P receptors consisting of 5 subtypes [5]. Three $\mathrm{S} 1 \mathrm{P}$ receptors, $\mathrm{S} 1 \mathrm{P} 1, \mathrm{~S} 1 \mathrm{P} 2$, and S1P3 receptors, are expressed in several cell types. S1P4 receptor is primarily expressed in cells of hematopoietic origin. S1P5 receptor is expressed in normal brain [6]. These S1P receptors regulate numerous downstream signaling pathways. These findings suggest a possibility that various effects of S1P may be attributed to the expression pattern of its receptors. Several cancer cells expressed S1P receptors. Breast cancer cells express S1P2 and S1P3 receptors [7]. Glioma cells express S1P1, S1P2, and S1P3 receptors [8], melanoma cells express S1P2 receptor [9]. In this study, we examined the expression of $\mathrm{S} 1 \mathrm{P}$ receptors (S1P1, S1P2, and S1P3) in malignant bone and soft tissue tumors. Furthermore Ki-67 is a nuclear antigen present in all cycling human cells and a marker of active cell proliferation, so we investigated all tumor sample cells proliferative activity as assessed by Ki-67. The first aim of this study is to examine the expression of $\mathrm{S} 1 \mathrm{P}$ receptors as a comparative study between high grade malignant tumors and low grade malignant tumors.

A major component in the biosynthesis of S1P is sphingosine kinase (SPHK). Both isoenzymes, SPHK1 and SPHK2, phosphorylate the precursor molecule sphingosine, producing S1P, and are the most essential elements in sphingolipid metabolism because they maintain the balance between apoptotic ceramide and anti- apoptotic S1P [10-13]. The molecular function of SPHK1 is known in several cells, and SPHK1 inhibits apoptosis and initiates cell growth. Although much less is known of the functions and regulation of SPHK2, recently it is suggested that SPHK2 may promote apoptosis and inhibit cell growth [14]. Roles of SPHK2 are still uncertain in normal or pathologic conditions. The expression of SPHK1 mRNA is elevated in many cancers [15]. The expression of SPHK1 in glioblastoma multiforme correlates with a shorter patient survival time [16]. In a recent study, it is suggested that SPHK1 expression is required for tumor cell proliferation [17]. The second aim of this study is to examine the expression of SPHK1 in malignant bone and soft tissue tumors and their correlation with histologic grade.

Overexpression of SPHK1 has been shown to enhance proliferation, and SPHK1 inhibitors decreased proliferation of malignant cell lines [18]. Recently SPHK1 has been cloned and has shown to be expressed in wide variety of tissues at various levels. It has been also demonstrated that SPHK1 may enhance cell proliferation. So we determined the role of SPHK1 in proliferation of malignant fibrous histiocytoma (MFH) cell lines. In this study, we examined the expressions of SPHK1 in MFH cell lines by Western blotting. We also tried to explore the role of SPHK1 in proliferation of MFH cell line by down-regulations of SPHK1. In malignant bone and soft tissue tumors, to our knowledge, this is the first study to examine the role of S1P receptors and SPHK.

\section{Materials and Methods}

\subsection{Tumor Samples}

Twenty-one tumor samples were obtained by open biopsy at Kobe University Hospital, and Hyogo Cancer Center in Japan. This study was conducted with the understanding and the consent of all patients before biopsy in accordance with institutional guide line. Tumor tissues were 7 liposarcomas, 3 chondrosarcomas (CS), 6 osteosarcomas (OS), 5 malignant fibrous histiocytoma (MFH). Four human MFH cell lines were used in this study (Nara H, Nara F, TNMY1, GBS-1). Nara H and Nara F were purchased from ScienStuff Co., Nara, Japan [19]. TNMY1 was established in our laboratory [20]. GBS-1 was kindly provided by Dr. H. Kanda (Department of Pathology, The Cancer Institute of the Japanese Foundation for Cancer Research, Tokyo, Japan) [21]. The cell lines were cultured in MEM medium containing 10\% FBS and antibiotics.

\subsection{Immunohistochemical Analysis}

Immunohistochemical staining for MIB-1 by Ki-67 antibody was performed for all malignant soft and bone tissue tumor samples. The areas in the tumors with the highest proliferative activity were selected and the MIB-1 labelled cells were counted.

\subsection{RT-PCR}

Total RNAs were isolated using an RNeasy Mini Kit ${ }^{\circledR}$ from tumor samples. RNA was converted to cDNA by reverse transcription, performed by incubating $1 \mu \mathrm{l}$ of RNA in a reaction mixture containing $0.5 \mathrm{mg} / \mathrm{ml}$ of oligo(dT) primer, Reverse Transcription $10 \times$ Buffer, 10 mM dNTP Mix and AMV Reverse Transcriptase. PCR was performed using a Perkin-Elmer DNA thermal cycler. Receptor S1P1, 3, 5 were examined by reverse transcription PCR. Primer sequences for S1P receptors were chosen the sequences described previously [22]. Sequences for the S1P receptors specific RNAs are as follows: S1P1, 5'-CCACAACGGGAGCAATAACT-3' and 5'-GTAAATGATGGGGTTGGTGC-3'; S1P2, 5'-CCAATACCTTGCTCTCTGGC-3' and 5'-CAGAAGGAGGATGCTGAAGG-3'; S1P3, 
5'-TCAGGGAGGGCAGT ATGTT-3' and

5'-CTGAGCCTTGAAGAGGATGG-3'. The samples were preheated at $94^{\circ} \mathrm{C}$ for $3 \mathrm{~min}$, then cycled 40 times at $94^{\circ} \mathrm{C}$ for $60 \mathrm{sec}, 58^{\circ} \mathrm{C}$ for $60 \mathrm{sec}$ and $72^{\circ} \mathrm{C}$ for $60 \mathrm{sec}$ and finally $72^{\circ} \mathrm{C}$ for $7 \mathrm{~min}$. GAPDH was used as an internal control for RNA integrity. RT-PCR products were run on $2 \%$ agarose gel, stained with ethidium bromide, and visualized by UV illumination.

\subsection{Real Time PCR}

Reactions were carried out in $2 \times$ PCR Master Mix (Applied Biosystems Inc., Foster City, CA, USA) in a total volume of $50 \mu \mathrm{l}$ containing $5 \mu \mathrm{l}$ of cDNA, $0.4 \mu \mathrm{M}$ of each PCR primer, $0.2 \mu \mathrm{M}$ of TaqMan probe with passive reference, and $25 \mu \mathrm{l}$ of PCR Master Mix. SPHK and $\beta$-actin genes were amplified in separate tubes in duplicate. Primer sequences and protocol for real time PCR were chosen according to the sequences and protocol described previously [23]. The amplification parameters were $50^{\circ} \mathrm{C}$ for 2 minutes, $95^{\circ} \mathrm{C}$ for 10 minutes, and 50 cycles at $95^{\circ} \mathrm{C}$ for 15 seconds and at $60^{\circ} \mathrm{C}$ for 1 minute. The following exonspanning primers and probes were used:

SPHK1, forward

5'-TTGAACCATTATGCTGGCTATGAG-3'; reverse 5'-CAGCAGGTTCATGGGTGACA-3'; probe

5'-FAM-ACGCTATTGCTGTGCCGCCG-TAMURA-3'; $\beta$-actin, forward

5'-AGAAAATCTGGCACCACACC-3'; reverse 5'-AGAGGCGT ACAGGGATAGCA-3'; probe

5'-FAM-ACCGCGAGAAGATGACCCAGATC

-TAMRA-3'. PCR products were measured using ABI

PRISM 7700 Sequence Detection System.

\subsection{Western Blotting}

We used 4 MFH cell lines to analyze SPHK1 proteins. After isolating proteins in cytoplasm, proteins were separated under reducing condition by electrophoresis on $12 \%$ polyacrylamide gel and transblotted electrically onto PVDF membrane. SPHK1 proteins were detected using mouse anti-human SPHK1 monoclonal antibody (Santa Cruz Inc., Santa Cruz, CA, USA).

\subsection{Down-Regulation of SPHK1}

SPHK1 siRNA (Ambion Inc., Austin, TX, USA) was transfected into MFH cells by lipofection method. Inhibition levels of SPHK1 mRNA were compared between SPHK1 siRNA and non specific control siRNA by real time PCR. Down-regulation of SPHK1 proteins was confirmed by Western blotting using SPHK1 specific anti- bodies.

\subsection{Cell Growth Analysis}

Cell proliferation was assayed using WST-8 (Cell Counting Kit-8) assay. Cells (control and transfected with siRNA) were trypsinized and seeded at a density of approximately 5000 cells/well in 96-well culture plates (used 60-wells, one outside circumference line did not use) in $100 \mu \mathrm{l}$ culture medium with $10 \%$ FBS. On each subsequent day, cells from 12 wells counted using Cell Counting Kit-8.

\subsection{Statistical Analysis}

A Chi square analysis was used to compare for categorical variables, Man-Whitney $U$ test and Student $t$ test analysis were used for continuous variables. Probability values less than 0.05 were considered to be significant.

\section{Results}

The median proliferative index (MIB-1 index) was 2.2\% (range of $1.1 \%-3.1 \%$ ) for liposarcoma, 6.4\% (range of $4.0 \%-10.1 \%$ ) for chondrosarcoma, $48 \%$ (range of $37 \%$ $52 \%$ ) for osteosarcoma, $35 \%$ (range of $24 \%-42 \%$ ) for MFH. Apparently high grade malignant tumors (OS and MFH) were stained by Ki-67 more than low grade malignant tumors (liposarcoma and CS). Therefore, we defined OS and MFH as high-grade tumor, whereas CS and liposarcoma as low-grade malignant tumor, and conducted further study.

By RT-PCR analysis, all high-grade malignant tumors (OS and MFH) expressed S1P1, S1P2, and S1P3 receptors (Figure 1). In low-grade malignant tumors (CS and liposarcoma), the expression of S1P1 receptor was detected in $50 \%, \mathrm{~S} 1 \mathrm{P} 2$ receptor in $30 \%$, and $\mathrm{S} 1 \mathrm{P} 3$ receptor in 50\% (Figure 2). The incidence of S1P1, S1P2, and S1P3 receptors expression between high-grade and low-grade malignant tumors was statistically different $(p<0.05)$.
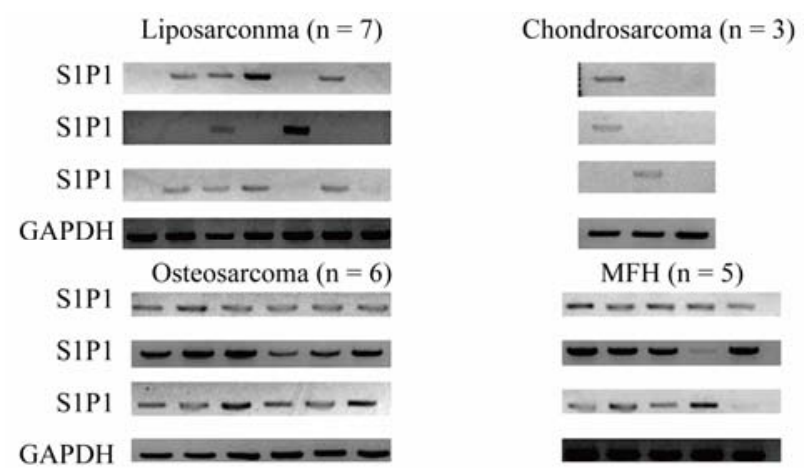

Figure 1. Gene expression of S1P receptors in tumor samples by RT-PCR. mRNA s of S1P1, S1P2 and S1P3 receptors were expressed in all osteosarcoma and MFH tissues, but not in all liposarcoma and chondrosarcoma tissues. 


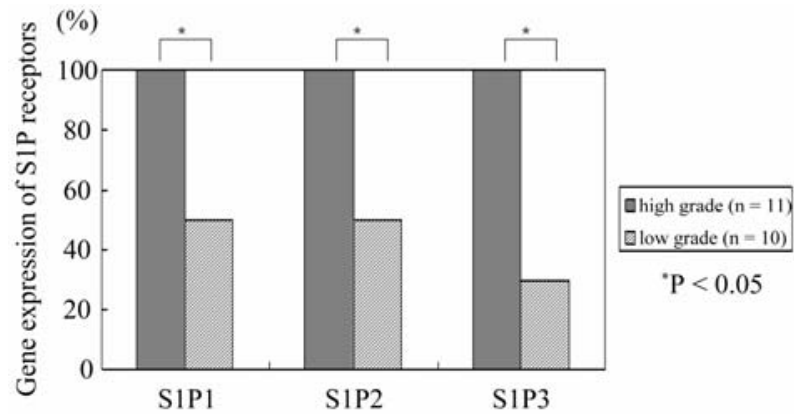

Figure 2. Expression rates of S1P receptors mRNA. All high-grade malignant tumors (OS and MFH) expressed S1P1, S1P2, and S1P3 receptors. Low-grade malignant tumors (CS and liposarcoma), the expression of S1P1 receptor was detected in $50 \%, \mathrm{~S} 1 \mathrm{P} 2$ receptor in $30 \%$, and $\mathrm{S} 1 \mathrm{P} 3$ receptor in $\mathbf{5 0 \%}$.

By real-time RT-PCR analysis, no statistically significant difference was found in the expression level of SPHK1 between high-grade and low-grade malignant tumors.

By results of Western blotting, proteins of SPHK1 were expressed in all MFH cell lines (Figure 3).

In MFH cell lines, transfection with SPHK1 siRNA oligonucleotides resulted in approximately $50 \%$ to $80 \%$ suppression of SPHK1 mRNA expression as determined by real-time PCR (Figure 4). As expected, down-regulation of SPHK1 with small interfering RNA significantly reduced SPHK1 protein levels by Western blotting (Figure 5). Knock down of SPHK1 expression significantly suppressed cell proliferation $(p<0.05)$ of all MFH cells between 3, 4 and 5 days after transfection with siRNA (Figure 6).

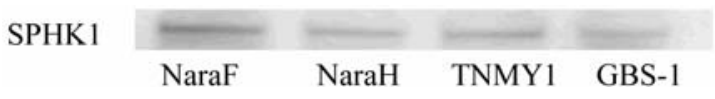

Figure 3. SPHK1 protein expressions of four human MFH cell lines by Western blotting. All MFH cell lines expressed SPHK1 proteins.

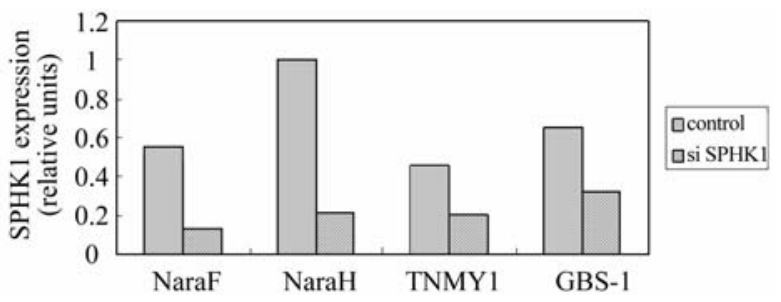

Figure 4. SPHK1 expression levels were quantified using real-time PCR (relative units). Transfection with SPHK1 siRNA resulted in $76 \%$ suppression of SPHK1 mRNA expression in NaraF cell line, $79 \%$ suppression in NaraH cell line, $55 \%$ suppression in TNYM1 cell line, $\mathbf{5 1 \%}$ suppression in GBS-1 cell line.

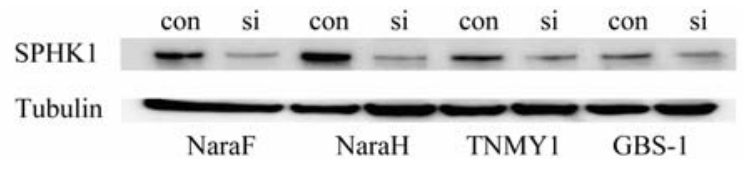

Figure 5. Cells were transfected with control siRNA(con) or siRNA(si) to SPHK1, and 2 days later, expression of SPHK1 proteins were measured by Western blotting analysis in 4 MFH cell lines. All MFH cell lines down-regulation of SPHK1 with siRNA significantly reduced SPHK1 protein levels.

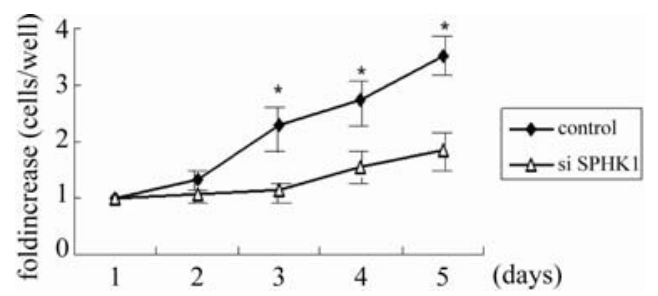

(a)

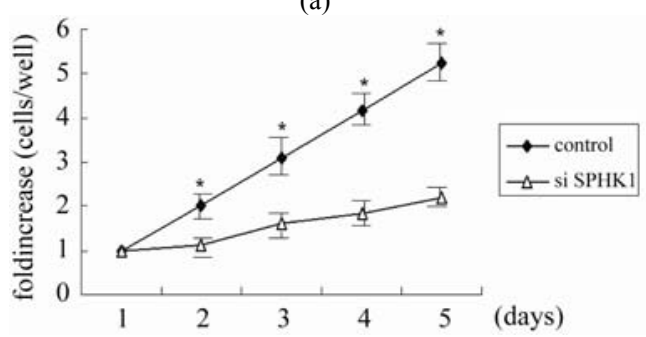

(b)

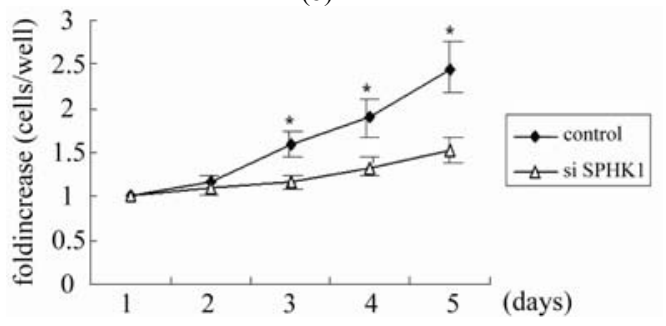

(c)

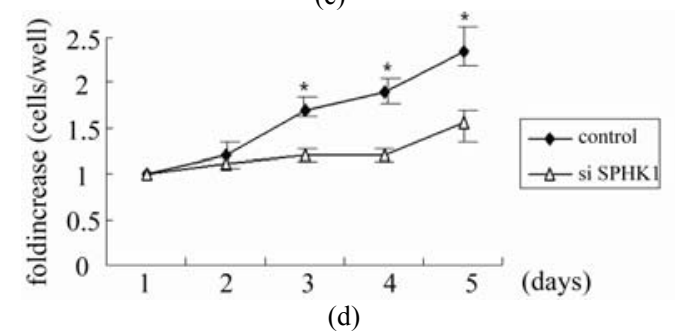

Figure 6. Growth curve analysis in 4 MFH cell lines. NaraF (a), NaraH (b), TNMY1 (c), GBS-1 (d) cells growth were measured by growth curve analysis as described in Materials and Methods. All SPHK1 knockdown data points at day 3 and beyond were significantly different from the control at the respective day (NaraH knockdown data point were significantly different from day 2). Data are expressed as fold increase in cell number over time relative to cells present at Day 1. The asterisks $(*)$ indicate statistically significant difference from control cells by Student $t$ test $(p<0.05)$. 


\section{Discussion}

$\mathrm{S} 1 \mathrm{P}$ is a bioactive lysophospholipid that mediates a variety of biological responses, including cell proliferation, survival and motility. S1P acts as an important intracellular mediator and an extracellular agonist. Currently, the roles of S1P signaling through S1P receptors have been shown to be important for cell activity [1-3]. S1P receptors have downstream effectors in common, yet there is enough variation among the receptors. S1P1 couples to $\mathrm{Gi}, \mathrm{S} 1 \mathrm{P} 2$ couples to $\mathrm{Gq}, \mathrm{G} 13$, and $\mathrm{Gi}$, similarly S1P3 couples to Gq, G13, and Gi. After activation of G-proteins, downstream cellular signaling pathways, such as protein phosphorlyation pathways, second messenger systems (cAMP and $\mathrm{Ca}^{++}$) are activated [24]. These intra-cellular signal pathways eventually change cell behavior such as gene transcription, cell morphology and movement. Previous studies have strongly suggested that extracellular bioactive lipids S1P and related ligands mediate many of their activation via the S1P1-5 GPCRs. It has been reported that many S1P receptors express in malignant cells. Glioma cells, breast cancer cells, gastric cancer cells express S1P receptors [7,8,25]. In the current study, all osteosarcoma cells and MFH cells (high grade malignant bone and soft tissue tumor cells) expressed S1P receptors. In low grade malignant tumors (CS and liposarcoma), the expression of S1P1 receptor is detected in $50 \%$, S1P2 receptor in $50 \%$, S1P3 receptor in $30 \%$. We can recognize that most malignant bone and soft tissue tumors express S1P receptors. The incidence of S1P1, $\mathrm{S} 1 \mathrm{P} 2$, and S1P3 receptors expression between high-grade and low-grade malignant tumors is statistically different. These results suggest that the expressions of S1P receptors may play an important role for cell proliferation, apoptosis, and may correlate with histologic grade in malignant bone and soft tissue tumors. Therefore, the expression patterns of S1P receptor may help to differentiate high grade malignant tumors from low grade malignant or benign tumors and to predict the prognosis of the patients with malignant bone and soft tissue tumors. Recently several studies have indicated that the S1Preceptor-agonist-analogue (FTY720) inhibits angiogenesis and tumor vascularization in vivo and suppresses tumor growth $[6,26]$. In the current study, all osteosarcoma and MFH cells expressed S1P receptors, therefore it is speculated that S1P receptor analogue could be a molecular-targeted drug for osteosarcoma and MFH. Further studies should be conducted to test that S1P-receptoragonist-analogue (FTY720 etc.) may inhibit cell proliferation and tumor growth of MFH in vitro and in vivo.

Sphingosine kinases (SPHK) catalyze the phosphorylation of sphingosine, thereby forming the S1P. Two dis- tinct SPHK isoforms, SPHK1 and SPHK2, have been characterized. Two isotypes of SPHK have shown to be expressed in a wide variety of tissues at various levels. Recently it is considered that SPHK1 has pro-survival functions and is mainly cytosolic protein. In contrast to SPHK1, SPHK2 may inhibit cell growth and enhances apoptosis [10-12]. Chronic regulation of SPHK1 at the transcriptional level is associated with cell proliferation and survival, up-regulation of SPHK1 occurs in a number of tumors. SPHK1 has been expressed in several forms of cancer. In breast cancer, it has been demonstrated that overexpression of SPHK1 enhanced proliferation, decreased apoptosis [27]. On the other hand, much less is known of the functions and regulation of SPHK2, it is considered that SPHK2 may suppress growth and enhance apoptosis, but the roles of SPHK2 are still unknown. In the current study, all bone and soft tissue malignant tumors expressed SPHK1 isoform. We could not find a statistically significant difference in the expression level of SPHK1 between high-grade and low-grade malignant tumors.

In malignant soft tissue tumors, malignant fibrous histiocytoma (MFH) is the most common primary tumor. MFH is clinically very aggressive and has a high metastatic potential. The prognosis is very poor. Therefore several chemotherapy protocols are currently used for $\mathrm{MFH}$, but don't sufficiently improve prognosis of the patients with MFH [28]. S1P is a bioactive lysophospholipid that mediates a variety of biological responses, including cell proliferation [1-4]. S1P is generated by phosphorylation of sphingosine catalyzed by SPHK1. Previous studies have confirmed that overexpression of SPHK1 and increased S1P production promotes cell growth [29-31]. SPHK1 is frequently overexpressed in a varietly of solid tumors, suggesting a potential oncogenic function and an important role in human tumorigenesis. Recently SPHK1 inhibitors have shown to decrease proliferation of cancer cell lines [32-36]. In the current study, we could confirm that all MFH cell lines expressed SPHK1 proteins by Western blotting. Furthermore we hypothesized that the down-regulations of SPHK1 might inhibit growth of MFH cells in culture. We could find that down-regulation of SPHK1 caused the suppression of cell proliferation in MFH cells. These results suggest that the specific inhibitors or gene therapies for SPHK1 can be useful therapeutic tools for MFH. Recently, the SPHK1-specific inhibitor (SK1-I) was developed and it has been demonstrated that SK1-I inhibited cell proliferation and tumor growth of leukemia and glioblastoma $[35,36]$. SK1-I may deserve a potent drug for malignant bone and soft tissue tumors. Further studies need to test the effects of SK1-I on cell proliferation and tumor 
growth of skeletal malignant tumors.

The limitations of this study are as follows. First, we evaluated roles of SIP as a extracellular messenger related to S1P receptors in the current study. However, S1P is also known to be an intracellular second messenger as shown in the previous studies. The roles of SIP as an intracellular second messenger are still unknown in bone and soft tissue tumors. Second, the current study did not evaluate the expression and roles of SPHK2 in bone and soft tissue tumors. Our preliminary results suggest that the expression level of SPHK2 in low-grade malignant tumors tends to be higher than that in high-grade malignant tumors. The roles of SPHK2 in bone and soft tissue tumors are still under-investigated in our in vitro and in vivo studies.

In conclusion, we have shown that $\mathrm{S} 1 \mathrm{P}$ receptors and SPHK1 express most malignant bone and soft tissue tumors, furthermore S1P receptor expression level may correlate with histologic grade in malignant bone and soft tissue tumors. Therefore, S1P receptor analogue could provide useful therapeutic tools targeted osteosarcoma and MFH in the future. SPHK1 inhibitors also might make useful therapeutic target in MFH cells.

\section{Acknowlegements}

The authors express their thanks to Ms. Janina Tubby for her editorial assistance.

\section{REFERENCES}

[1] T. Hla, "Signaling and Biological Actions of Sphingosine 1-phosphate," Pharmacological Research, Vol. 47, No. 5, 2003, pp. 401-407. doi:10.1016/S1043-6618(03)00046-X

[2] S. E. Alvarez, S. Milstien and S. Spiegel, "Autocrine and Paracrine Roles of Sphingosine-1-phosphate," Trends in Endocrinology \& Metabolism, Vol. 18, No. 8, 2007, pp. 300-307. doi:10.1016/j.tem.2007.07.005

[3] Y. A. Hannun and L. M. Obeid, "Principles of Bioactive Lipid Signalling: Lessons from Sphingolipids," Nature Reviews Molecular Cell Biology, Vol. 9, No. 2, 2008, pp.139-150. doi:10.1038/nrm2329

[4] T. C. Pham, J. I. Sr. Fells, D. A. Osborne, E. J. North, M. M. Naor and A. L. Parrill, "Molecular Recognition in the Sphingosine-1-phosphate Receptor Family," Journal of Molecular Graphics and Modeling, Vol. 26, No. 8, 2008, pp. 1189-1201. doi:10.1016/j.jmgm.2007.11.001

[5] T. A. Taha, K. M. Argraves and L. M. Obeid, "Sphingosine-1-phosphate Receptors: Receptor Specificity Versus Functional Redundancy," Biochimica et Biophysica Acta, Vol. 1682, No. 1-3, 2004, pp. 48-55.

[6] V. Brinkmann, "Sphingosine-1-phosphate Receptors in Health and Disease: Mechanistic Insights from Gene Deletion Studies and Reverse Pharmacology," Pharmacology \& Therapeutics, Vol. 115, No. 1, 2007, pp. 84-105.

\section{doi:10.1016/j.pharmthera.2007.04.006}

[7] E. J. Goetzl, H. Dolezalova, Y. Kong and L. Zeng, "Dual Mechanisms for Lysophospholipid Induction of Proliferation of Human Breast Carcinoma Cells," Cancer Research, Vol. 59, No. 18, 1999, pp. 4732-4737.

[8] J. R. Van Brocklyn, N. Young and R. Roof, "Sphingosine-1-phosphate Stimulates Motility and Invasiveness of Human Glioblastoma Multiforme Cell," Cancer Letters, Vol. 199, No. 1, 2003, pp. 53-60.

doi:10.1016/S0304-3835(03)00334-3

[9] H. Yamaguchi, J. Kitayama, N. Takuwa, K. Arikawa, I. Inoki, K. Takehara, H. Nagawa and Y. Takuwa, "Sphingosine-1-phosphate Receptor Subtype-Specific Positive and Negative Regulation of Rac and Haematogenous Metastasis of Melanoma Cells," Biochemical Journal, Vol. 374, No. 3, 2003, pp. 715-722. doi:10.1042/BJ20030381

[10] N. C. Hait, C. A. Oskeritzian, S. W. Paugh, S. Milstien and S. Spiegel, "Sphingosine Kinases, Sphingosine1-phosphate, Apoptosis and Diseases," Biochimica et Biophysica Acta, Vol. 1758, No. 12, 2006, pp. 20162026. doi:10.1016/j.bbamem.2006.08.007

[11] D. R. Gude, S. E. Alvarez, S. W. Paugh, P. Mitra P, J. Yu, R. Griffiths, S. E. Barbour, S. Milstien and S. Spiegel, "Apoptosis Induces Expression of Sphingosine Kinase 1 to Release Sphingosine-1-phosphate as a "Come-andGet-Me' Signal," The FASEB Journal, Vol. 22, No. 8, 2008, pp. 2629-2638. doi:10.1096/fj.08-107169

[12] S. Pyne, S. C. Lee, J. Long and N. J. Pyne, "Role of Sphingosine Kinases and Lipid Phosphate Phosphatases in Regulating Spatial Sphingosine-1-phosphate Signalling in Health and Disease," Cell Signal, Vol. 21, No. 1, 2009 , pp. 14-21. doi:10.1016/j.cellsig.2008.08.008

[13] A. J. Melendez, "Sphingosine Kinase Signalling in Immune Cells: Potential as Novel Therapeutic Targets," Biochimica et Biophysica Acta, Vol. 1784, No. 1, 2008, pp. 66-75.

[14] M. Maceyka, H. Sankala, N. C. Hait, H. Le Stunff, H. Liu, R. Toman, C. Collier, M. Zhang, L. S. Satin, A. H. Merrill Jr., S. Milstien and S. Spiegel, "SPHK1 and SPHK2, Sphingosine Kinase Isoenzymes with Opposing Functions in Sphingolipid Metabolism," The Journal of Biological Chemistry, Vol. 280, No. 44, 2005, pp. 37118-37129. doi:10.1074/jbc.M502207200

[15] M. Vadas, P. Xia, G. McCaughan and J. Gamble, "The Role of Sphingosine Kinase 1 in Cancer: Oncogene or Non-Oncogene Addiction?" Biochimica et Biophysica Acta, Vol. 1781, No. 9, 2008, pp. 442-447.

[16] J. R. van Brocklyn, C. A. Jackson, D. K. Pearl, M. S. Kotur, P. J. Snyder and T. W. Prior, "Sphingosine Kinase-1 Expression Correlates with Poor Survival of Patients with Glioblastoma Multiforme: Roles of Sphingosine Kinase Isoforms in Growth of Glioblastoma Cell Lines," Journal of Neuropathology \& Experimental Neurology, Vol. 64, No. 8, 2005, pp. 695- 705. doi:10.1097/01.jnen.0000175329.59092.2c 
[17] R. Alemany, C. J. van Koppen, K. Danneberg, M. Ter Braak and D. Meyer zu Heringdorf, "Regulation and Functional Roles of Sphingosine Kinases," Naunyn-Schmiedeberg's Archives of Pharmacology, Vol. 374, No. 5-6, 2007, pp. 413-428. doi:10.1007/s00210-007-0132-3

[18] C. Ricci, F. Onida, F. Servida, F. Radaelli, G. Saporiti, K. Todoerti, G. L. Deliliers and R. Ghidoni, "In Vitro Anti-Leukaemia Activity of Sphingosine Kinase Inhibitor," British Journal of Haematology, Vol. 144, No. 3, 2009, pp. 350-357. doi:10.1111/j.1365-2141.2008.07474.x

[19] Y. Kiyozuka, H. Nakagawa, Y. Uemura, H. Senzaki, A. Yamamoto, T. Noguchi, H. Mizuta, K. Nakanishi, S. Nakano and A. Tsubura, "Novel Cell Lines Established from a Human Myxoid Malignant Fibrous Histiocytoma Arising in the Uterus," Cancer Genetics and Cytogenetics, Vol. 127, No. 1, 2001, pp. 7-15. doi:10.1016/S0165-4608(00)00413-1

[20] T. Nakatani, T. Marui, T. Yamamoto, M. Kurosaka, T. Akisue and K. Matsumoto, "Establishment and Characterization of Cell Line TNMY1 Derived from Human Malignant Fibrous Histiocytoma," Pathology International, Vol. 51, No. 8, 2001, pp. 595-602. doi:10.1046/j.1440-1827.2001.01253.x

[21] Z. Fang, H. Mukai, K. Nomura, K. Shinomiya, S. Matsumoto, N. Kawaguchi, T. Kitagawa and H. Kanda, "Establishment and Characterization of a Cell Line from a Malignant Fibrous Histiocytoma of Bone Developing in a Patient with Multiple Fibrous Dysplasia," Journal of Cancer Research and Clinical Oncology, Vol. 128, No. 1, 2002, pp. 45-49. doi:10.1007/s00432-001-0295-0

[22] C. Hornuss, R. Hammermann, M. Fuhrmann, U. R. Juergens and K. Racké, "Human and Rat Alveolar Macrophages Express Multiple EDG Receptors," European Journal of Pharmacology, Vol. 429, No. 1-3, 2001, pp. 303-308. doi:10.1016/S0014-2999(01)01329-2

[23] X. Pi, S. Y. Tan, M. Hayes, L. Xiao, J. A. Shayman, S. Ling and J. Holoshitz, "Sphingosine Kinase 1-Mediated Inhibition of Fas Death Signaling in Rheumatoid Arthritis B Lymphoblastoid Cells," Arthritis \& Rheumatism, Vol. 54, No. 3, 2006, pp. 754-764. doi:10.1002/art.21635

[24] S. Pyne and N. Pyne, "Sphingosine-1-phosphate Signalling via the Endothelial Differentiation Gene Family of G-Protein-Coupled Receptors," Pharmacology \& Therapeutics, Vol. 88, No. 2, 2000, pp. 115-131. doi:10.1016/S0163-7258(00)00084-X

[25] H. Yamashita, J. Kitayama, D. Shida, H. Yamaguchi, K. Mori, M. Osada, S. Aoki, Y. Yatomi, Y. Takuwa and H. Nagawa, "Sphingosine-1-phosphate Receptor Expression Profile in Human Gastric Cancer Cells: Differential Regulation on the Migration and Proliferation," Journal of Surgical Research, Vol. 130, No. 1, 2006, pp. 80-87. doi:10.1016/i.jss.2005.08.004

[26] M. Murph and G. B. Mills, "Targeting the Lipids LPA and S1P and Their Signalling Pathways to Inhibit Tumour Progression," Expert Reviews in Molecular Medicine, Vol. 9, No. 28, 2007, pp. 1-18. doi:10.1017/S1462399407000476

[27] S. Sarkar, M. Maceyka, N. C. Hait, S. W. Paugh, H. San- kala, S. Milstien and S. Spiegel, "Sphingosine Kinase 1 is Required for Migration, Proliferation and Survival of MCF-7 Human Breast Cancer Cells," FEBS Letters, Vol. 579, No. 24, 2005, pp. 5313-5317. doi:10.1016/j.febslet.2005.08.055

[28] S. W. Weiss and J. R. Goldblum, "Enzinger \& Weiss's Soft Tissue Tumors," Mosby Inc., Louisville, 2008. doi:10.1158/1078-0432.CCR-08-1158

[29] W. Li, C. P. Yu, J. T. Xia, L. Zhang, G. X. Weng, H. Q. Zheng, Q. L. Kong, L. J. Hu, M. S. Zeng, Y. X. Zeng, M. Li, J. Li and L. B. Song, "Sphingosine Kinase 1 is Associated with Gastric Cancer Progression and Poor Survival of Patients," Clinical Cancer Research, Vol. 15, No. 4, 2009, pp. 1393-1399.

[30] T. Kawamori, T. Kaneshiro, M. Okumura, S. Maalouf, A. Uflacker, J. Bielawski, Y. A. Hannun and L. M. Obeid, "Role for Sphingosine Kinase 1 in Colon Carcinogenesis," The FASEB Journal, Vol. 23, No. 2, 2009, pp. 405-414. doi:10.1096/fj.08-117572

[31] J. Li, H. Y. Guan, L. Y. Gong, L. B. Song, N. Zhang, J. Wu, J. Yuan, Y. J. Zheng, Z. S. Huang and M. Li, "Clinical Significance of Sphingosine Kinase-1 Expression in Human Astrocytomas Progression and Overall Patient Survival," Clinical Cancer Research, Vol. 14, No. 21, 2008, pp. 6996-7003. doi:10.1158/1078-0432.CCR-08-0754

[32] K. J. French, J. J. Upson, S. N. Keller, Y. Zhuang, J. K. Yun and C. D. Smith, "Antitumor Activity of Sphingosine Kinase Inhibitors," Journal of Neuropathology \& Experimental Neurology, Vol. 318, No. 2, 2006, pp. 596-603.

[33] K. J. French, R. S. Schrecengost, B. D. Lee, Y. Zhuang, S. N. Smith, J. L. Eberly, J. K. Yun and C. D. Smith, "Discovery and Evaluation of Inhibitors of Human Sphingosine Kinase," Cancer Research, Vol. 63, No. 18, 2003, pp. 5962-5969.

[34] M. E. Leroux, E. Auzenne, R. Evans, N. Jr. Hail, W. Spohn, S. C. Ghosh, D. Farquhar, T. McDonnell and J. Klostergaard, "Sphingolipids and the Sphingosine Kinase Inhibitor, SKI II, Induce BCL-2-Independent Apoptosis in Human Prostatic Adenocarcinoma Cells," Prostate, Vol. 67, No. 15, 2007, pp. 1699-1717. doi: $10.1002 /$ pros. 20645

[35] S. W. Paugh, B. S. Paugh, M. Rahmani, D. Kapitonov, J. A. Almenara, T. Kordula, S. Milstien, J. K. Adams, R. E. Zipkin, S. Grant and S. Spiegel, "A Selective Sphingosine Kinase 1 Inhibitor Integrates Multiple Molecular Therapeutic Targets in Human Leukemia," Blood, Vol. 112, No. 4, 2008, pp. 1382-1391. doi:10.1182/blood-2008-02-138958

[36] D. Kapitonov, J. C. Allegood, C. Mitchell, N. C. Hait, J. A. Almenara, J. K. Adams, R. E. Zipkin, P. Dent, T. Kordula, S. Milstien and S. Spiegel, "Targeting Sphingosine Kinase 1 Inhibits Akt Signaling, Induces Apoptosis, and Suppresses Growth of Human Glioblastoma Cells and Xenografts," Cancer Research, Vol. 69, No. 17, 2009, pp. 6915-6923. doi:10.1158/0008-5472.CAN-09-0664 